\title{
Project SUMS (scaling up of mental health in schools): design and methods for a pragmatic, cluster randomised waitlist- controlled trial on integrated school mental health intervention for adolescents
}

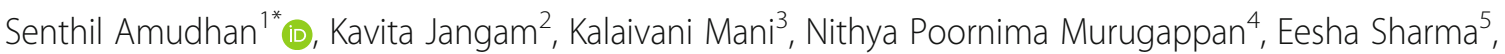

Payel Mahapatra', Ajit Deo Burma ${ }^{1}$, Hemant Kumar Tiwari ${ }^{1}$, Ashi Ashok', Sangappa Vaggar ${ }^{1}$ and

Girish Nagaraja Rao ${ }^{1}$

\begin{abstract}
Background: There is an increasing need for Mental Health Promotion (MHP) among adolescents, especially in developing countries with limited resources and rapid socio-demographic transition. With the growing burden of mental health problems among adolescents (suicide, depression) and their preferences to seek help from their peers, improving Mental Health Literacy (MHL) and behaviours for First Aid in Mental Health (MH-FA) becomes crucial to promote their mental health.

Methods: Schools are ideal settings for reaching the vulnerable adolescents. The proposed study evaluates the effectiveness of a classroom-based teacher-led integrated school mental health intervention called SUMS (MHP + $\mathrm{MHL}+\mathrm{MH}-\mathrm{FA}$ ). The study will involve a pragmatic, cluster-randomised waitlist-controlled design to evaluate the effectiveness of SUMS intervention using schools as unit-of-randomisation. The study will be conducted in Srinivaspura taluka (Sub-district) of Kolar district (administrative unit of health) of Karnataka in collaboration with a multi-disciplinary expert team from NIMHANS (National Institute of Mental Health And Neuro Sciences), Bangalore-India and Department of Education, Government of Karnataka, India. A total of 8 schools (400 students studying in 6-8 grade) from Srinivaspura taluka will be randomised into intervention and waitlist control group. The intervention group will receive SUMS intervention through 10-15 h of classroom sessions. The primary outcome is the improvement in positive mental health literacy, as measured by the Mental Health-Promoting Knowledge (MHPK-10) scale. Changes in MH-FA knowledge and intentions, Mental health stigma, help-seeking and resilience are assessed as secondary outcomes. Data will be collected at baseline, 6-weeks, 6-months and 12-months post-intervention. The waitlist-control schools will receive the interventions at the end of the 12-month follow-up assessment in intervention-schools.
\end{abstract}

\footnotetext{
* Correspondence: sam_mmc1999@yahoo.co.in

'Department of Epidemiology, National Institute of Mental Health And Neuro Sciences, Bangalore, Karnataka, India

Full list of author information is available at the end of the article
}

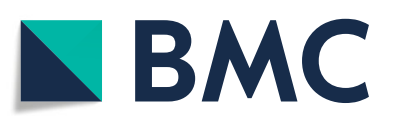

(- The Author(s). 2021 Open Access This article is licensed under a Creative Commons Attribution 4.0 International License, which permits use, sharing, adaptation, distribution and reproduction in any medium or format, as long as you give appropriate credit to the original author(s) and the source, provide a link to the Creative Commons licence, and indicate if changes were made. The images or other third party material in this article are included in the article's Creative Commons licence, unless indicated otherwise in a credit line to the material. If material is not included in the article's Creative Commons licence and your intended use is not permitted by statutory regulation or exceeds the permitted use, you will need to obtain permission directly from the copyright holder. To view a copy of this licence, visit http://creativecommons.org/licenses/by/4.0/. The Creative Commons Public Domain Dedication waiver (http://creativecommons.org/publicdomain/zero/1.0/) applies to the data made available in this article, unless otherwise stated in a credit line to the data. 
Discussion: This is the first study to integrate Mental Health Literacy with Mental Health Promotion and behaviours for First Aid in Mental Health to promote mental health well-being among adolescent school children in India. With a need to build a more substantial evidence base on School Mental Health Promotion approaches in developing countries, the study findings will have implications for implementing and operationalising Health and Wellness Ambassador initiative in India.

Trial registration: Clinical Trials Registry - India, CTRI/2019/07/020394. Registered prospectively on 29 July 2019. (ctri. nic.in/Clinicaltrials/pmaindet2.php?trialid=35724\&EncHid=\&userName=sums).

Keywords: Mental health literacy, Mental health promotion, First aid in mental health, School mental health, Resilience, Cluster randomised trial

\section{Background}

In recent years, there is an increasing concern about adolescents' mental health, especially in developing countries. An estimated $10-20 \%$ of children and adolescents are affected by mental health problems worldwide [1]. Adolescence is a period that is characterised by rapid physical and psychological changes with altered social perceptions and expectations [2]. The expectations and the choices made during this period have a significant impact not only on current health-practices and wellbeing but also on their health as adults. We must engage with them and enable them to make right choices about their expectations and address their mental health needs effectively through mental health promotion (MHP). With rapid socio-demographic and lifestyle transition, such mental health promotion will help adolescents build resilience to cope well in difficult situations or adversities. This will be critical not only for their wellbeing during adolescence but also for their physical and mental health in adulthood.

Today, suicide is one of the leading cause of preventable deaths, and depression is one of the leading cause of morbidity among adolescents [3]. Based on the life-course perspective, it has been emphasised to target adolescents in school to accommodate the contextual factors that influence suicide during adolescence [4]. Even though these mental health problems have detrimental effects on wellbeing, and development in adolescence, a reluctance to seek professional help due to stigma and lack of mental health knowledge has been documented in various studies across the globe [5-7]. Thus, there is a need to improve help-seeking to reduce the growing burden of mental health problems among adolescents and promote mental health literacy as an important strategy to promote helpseeking among adolescents [8-10].

Mental health literacy (MHL) is defined as "knowledge and attitudes regarding mental health that aid in recognition, management and prevention of mental health issues" [11]. Low levels of MHL were associated with depression and MHL among adolescents was positively associated with their mental health $[12,13]$. Even though mental health literacy has significant implications on adolescent mental health, poor mental health literacy has been demonstrated for adolescents across various settings [14, 15]. Thus, MHL must become a focus of mental health interventions for adolescents. With the adolescents preferring to disclose mental illness symptoms to their peers and reluctant to seek professional help, the importance of improving behaviours for First Aid in Mental Health (MH-FA) to facilitate social support and help-seeking for mental illness cannot be overlooked. Mental health first aid is the help provided to a person who is developing a mental health problem, experiencing a worsening of an existing mental health problem or in a mental health crisis [16]. As adolescents begin to make decisions about their health and often have a preference for seeking help from peers, mental health literacy to adolescents should be provided ideally as a part of the school curriculum and not as ad-hoc events that occur infrequently (such as mental health parades, campaigns etc.,) [8]. It is further emphasised that schools should promote not only positive mental health but also MHL to enable students to understand mental health problems, reduce mental health stigma, promote mental health self-care and help-seeking behaviours of students [13]. Thus, integration of First Aid in Mental Health (MH-FA) with MHP and MHL, becomes a potential strategy for mental health promotion and prevention in adolescents.

Given the critical role of education in health literacy, schools become an ideal setting for reaching vulnerable adolescents for improving mental health literacy. This is because adolescents spend most of their time in schools, and health is intrinsically linked with education. Furthermore, many risks and protective factors for adverse mental health outcomes are operated in the school context. Presently, the evidence base for educational interventions targeting adolescents for mental health literacy and mental health promotion is limited [17]. Even though there is a concept of Health Promoting Schools (HPS) by World Health Organization that provides a broad framework for action, there is little evidence on 
schools' ability to implement this approach. Existing evaluations of schools that had implemented HPS initiative have shown that mental health (despite being an important outcome of HPS initiative) was not addressed, apart from substance use $[18,19]$. A clear need for wellcontrolled research in the area of mental health literacy and mental health promotion has been documented due to previous studies with small sample sizes, lack of follow-up, low response rates and potential contamination [20].

In India, adolescent mental health assumes a great relevance where adolescents aged $10-19$ years constitute about $22 \%$ of the population [21]. With increasing enrolment in secondary schools in India, schools become the ideal platform for promoting positive mental health among adolescents. Even though there are Adolescence Education Programme and Rashtriya Kishor Swasthya Karyakram programme in India, it was evident that such programmes have either failed to account for the mental health needs of school communities or failed to measure the changes or values that are intended for the sustenance of mental health promotion [22, 23].

Developing personal skills for both students and teachers has been emphasised for successful mental health interventions [13] Among various approaches to address mental health literacy in schools, a natural approach builds on schools' existing social ecologies such as curriculum presented by usual classroom teachers. Also, teacher-led approaches were found to facilitate the creation of a school-wide environment of acceptance and normalisation of mental illnesses as well as facilitate recognition and help-seeking in adolescents [8]. MHL delivered by external providers had limited value on the sustained and enhanced MHL capacity embedded into educational systems [13]. Further, MHL delivered by teachers was found to have positive mental health literacy outcomes across various settings and teacher delivered MHL curriculum found to have an advantage in normalising mental health knowledge as a part of education [13].

With this in the background, we developed a classroom-based teacher-led integrated school mental health intervention called SUMS (MHP $+\mathrm{MHL}+\mathrm{MH}-$ FA) based on "School Friendly" mental health literacy approach and "School-Based Integrated Pathway to Care Model" developed by Kutcher et al. [24]. Like MHL approach, SUMS intervention is based on existing classroom friendly pedagogical approach that can be easily delivered in the current educational systems without extracurricular or outside-of-school inputs [13]. Basically, SUMS intervention focuses on curriculum resource approaches delivered by teachers (https://mhlcurriculum. org/) where a curriculum resource guide was developed through desk review and expert inputs. The curriculum resource guide contained content and delivery methods for each SUMS intervention (MHL, MHP and MH-FA). This curriculum resource guide was adapted to the local context through key informant interviews and intervention designing workshops conducted among school teachers, students, block education officers and subject experts.

In this context, we have undertaken a cluster randomised controlled trial to evaluate the effectiveness of SUMS (Scaling Up of Mental Health in Schools) intervention (an integrated school mental health intervention) for adolescents, integrated into the classroom and delivered by teachers. We hypothesise that SUMS intervention delivered by teachers will lead to significant improvement in mental health knowledge, positive attitudes toward mental illness, and behaviours for First Aid in Mental Health as compared to ongoing regular school approach among school-going adolescent children. The ongoing regular school approach was used as comparator to reflect reallife scenario.

\section{Objectives}

This study's primary objective is to assess the effectiveness of evidence-informed integrated school mental health intervention (SUMS) in promoting mental health knowledge, positive attitudes toward mental illness, and behaviours for First Aid in Mental Health among adolescent school children. Furthermore, we will explore SUMS intervention's potential influence on a range of adolescent health-related outcomes such as academic achievement, absenteeism rate, educational stress, suicidal ideation, bullying, and substance use. Additionally, we will explore the implementation process of the intervention and identify contextual factors associated with outcomes.

\section{Methods \\ Study design}

The study will use a pragmatic, cluster randomised waitlist-controlled design with schools as unit-ofrandomisation to evaluate the effectiveness of SUMS intervention. The study will follow consort guidelines for cluster randomised trial (Fig. 1).

\section{Study area}

The study will be conducted in Srinivaspura taluka (Subdistrict) of Kolar district (administrative unit of health) of Karnataka (https://kolar.nic.in/en/) in collaboration with a multi-disciplinary expert team from NIMHANS (National Institute of Mental Health And Neuro Sciences), Bangalore-India and Department of Education, Government of Karnataka, India. Kolar district is the Public Health Observatory for Department of Epidemiology, NIMHANS. 


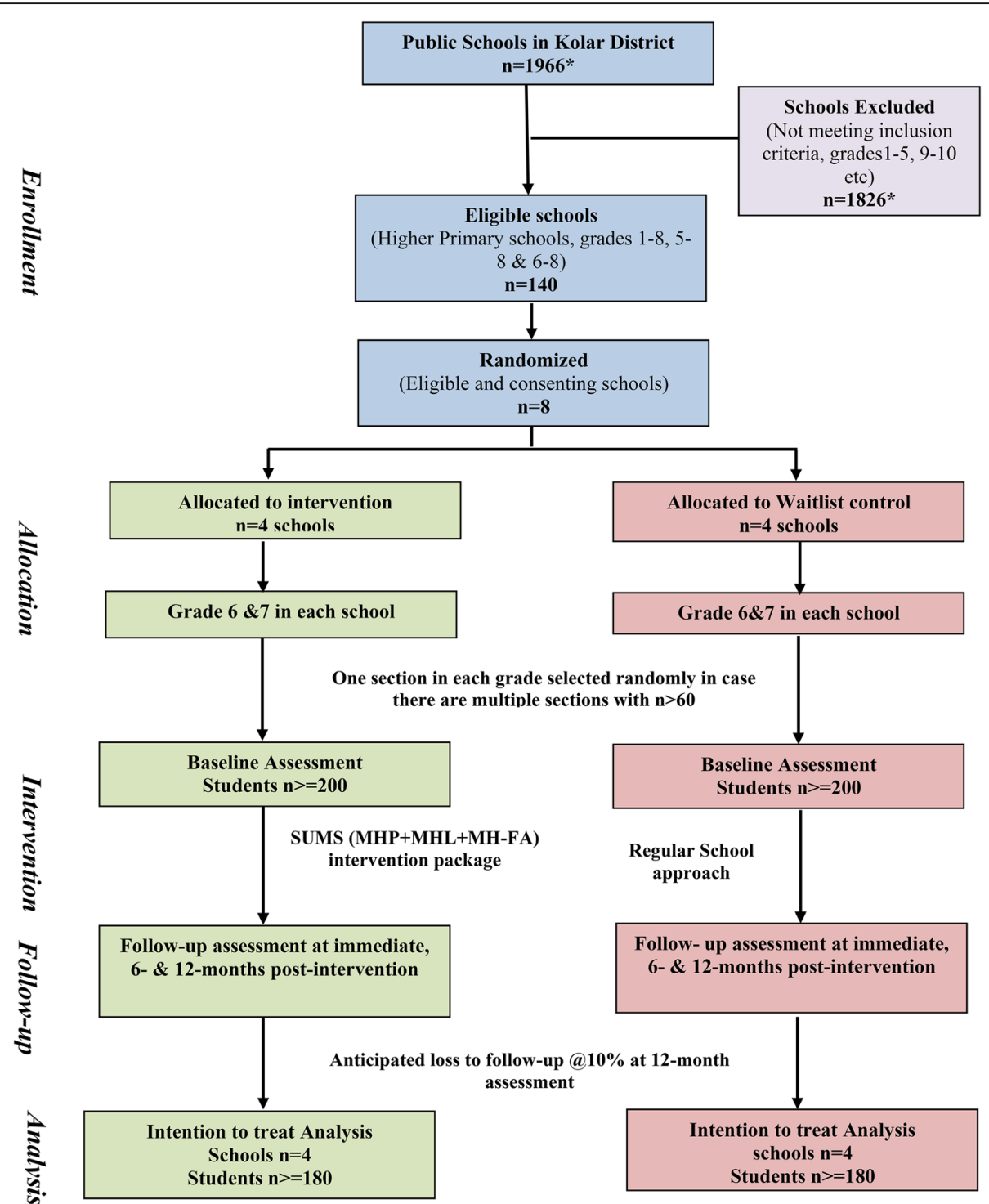

Fig. 1 Trial Design. MHP: Mental Health Promotion. MHL: Mental Health Literacy. MH-FA: First Aid in Mental Health. * Data obtained from Department of Public Instruction Office, Kolar for the year 2016

\section{Study setting}

The Department of Education in Karnataka state has three levels of schools viz., Primary (1-5 grades), Higher-Primary (6-8 grades) and High-schools (9-10 grades). The study will be conducted in higherprimary public schools in Srinivaspura taluka of Kolar District, Karnataka. Higher-primary public schools usually include children from ages 13 to 15 years. These grades were chosen because this is the period where life skills curriculum were introduced and this period in the life span also represents the point of departure for the rapid increase in psychiatric diagnoses that occurs prior to age 25 years [24].

\section{Study subjects}

The students from grades 6 and 7 will be included for the present study so that the students in grade- 7 will have 12-month follow-up when they are in class-8.

\section{Inclusion criteria}

All children enrolled in class 6th and 7th grade of the participating higher-primary public schools will be eligible for the present study.

\section{Exclusion criteria}

(1) Children who did not give informed assent (2) Children whose parents/guardians did not provide written informed consent (3) Children who were absent for the 
baseline assessment even after two attempted visits (4) children aged $>15$ years at enrolment.

\section{Intervention}

SUMS intervention (MHP + MHL + MH-FA) is a classroom-based teacher-led integrated school mental health intervention that will promote positive mental health, mental health literacy and behaviours for First Aid in Mental Health among school-going adolescent school children. As described earlier, SUMS intervention takes the form of a classroom-ready resource (the curriculum resource guide) designed to be delivered by usual classroom teachers to students in grades 6 and 7 (ages 12 to 15 years). The curriculum resource guide contains 10 modules categorised under 3 units (Mental Health Promotion, Mental Health Literacy and First Aid in Mental Health). The ten modules are as follows: Positive mental health, Promoting Resilience, Promoting Mindfulness, Life Skills Education, Mental Health Literacy, Understanding Mental Health and Mental Illness, Stigma of Mental Illness, Information on specific mental illnesses, seeking help and finding support, First Aid in Mental health and strategy for mental health first Aid (Table 1). Each module's purpose is to provide teachers with classroom-ready lesson plans, activities, and easily accessible resources. The ten modules are designed to be taught in sequence and are intended to be delivered on the usual school days through $10-15 \mathrm{~h}$ of classroom time.

Table 1 SUMS Intervention-Curriculum framework

\begin{tabular}{|c|c|c|}
\hline Units & Module & Teaching method \\
\hline $\begin{array}{l}\text { Mental Health } \\
\text { Promotion } \\
(\mathrm{MHP})\end{array}$ & $\begin{array}{l}\text { Module 1: Positive } \\
\text { mental health } \\
\text { Module 2: Promoting } \\
\text { Resilience } \\
\text { Module 3: Promoting } \\
\text { Mindfulness } \\
\text { Module 4: Life Skills } \\
\text { Education }\end{array}$ & \multirow[t]{3}{*}{$\begin{array}{l}\text { Educational session followed } \\
\text { by Participatory and } \\
\text { experiential activities as } \\
\text { recommended in the } \\
\text { resource guide (Interactive } \\
\text { discussions, Group discussion, } \\
\text { Co-operative play, Storytell- } \\
\text { ing. Role play, Brainstorming, } \\
\text { Debate, within-school com- } \\
\text { petitions, wall-magazine, etc.) }\end{array}$} \\
\hline $\begin{array}{l}\text { Mental Health } \\
\text { Literacy }(\mathrm{MHL})\end{array}$ & $\begin{array}{l}\text { Module 5: } \\
\text { Understanding Mental } \\
\text { Health and Mental } \\
\text { Illness } \\
\text { Module 6: Stigma of } \\
\text { Mental IIlness } \\
\text { Module 7: Information } \\
\text { on specific mental } \\
\text { illnesses } \\
\text { Module 8: Seeking help } \\
\text { and finding support }\end{array}$ & \\
\hline $\begin{array}{l}\text { First Aid in } \\
\text { Mental Health } \\
(\mathrm{MH}-\mathrm{FA})\end{array}$ & $\begin{array}{l}\text { Module 9: First Aid in } \\
\text { Mental health } \\
\text { Module 10: Strategy for } \\
\text { First Aid in Mental } \\
\text { health }\end{array}$ & \\
\hline
\end{tabular}

\section{Feasibility testing for SUMS interventions}

The SUMS intervention curriculum resource guide will be translated into local language Kannada and will be piloted with concerned Block Education Officers for its clarity and suitability for training school teachers and students. Perceived intervention feasibility will be assessed by adapting a 4-item Feasibility of Intervention Measure (FIM). Any suggestions by Block Education Officers will be duly accommodated to improve the feasibility [25].

\section{Outcomes}

The following core outcomes will be used to assess the intervention. All outcome measures will be measured at the individual student level.

\section{Primary outcome}

The primary outcome, Positive mental health literacy will be measured using a 10-item Mental HealthPromoting Knowledge (MHPK-10) scale. It is a valid and reliable one-dimensional instrument measuring knowledge of factors promoting good mental health among adolescents. The MHPK-10 had demonstrated good internal validity and test-retest reliability $(r=0.74)$ among adolescents. For each item, respondents rate items on 1-5 likert-type scale, the extent to which they are correct or wrong. The score range is $10-50$, with a higher score indicating a higher positive mental health literacy [26].

\section{Secondary outcomes}

Mental health first aid knowledge and mental health literacy This will be measured using a 35-item univariate scale, Mental Health Literacy Scale (MHLS). The scale will be adapted for use among adolescents. The score range is $35-160$, with a higher score indicating a higher mental health literacy [27].

Personal and public stigma towards mental health among adolescents This will be measured using the 24item Peer Mental Health Stigmatization Scale (PMHSS). For the present study, the 8-item personal stigma subscale will be used. The scale demonstrated adequate discriminant validity, internal consistency $(\alpha=0.75)$ and test-retest reliability in adolescents [28].

Stigmatising behavior will be measured using the Reported and Intended Behaviour Scale (RIBS). Scores on the RIBS range from 4 to 20 , with higher scores indicating more positive attitudes. The RIBS has a test-retest reliability of 0.75 , and a Cronbach's $\alpha$ of 0.86 [29].

Mental health help-seeking This will be assessed using a 24-item Inventory of Attitudes towards Seeking Mental 
Health Services (IASMHS) scale. It consists of three subscales; Psychological Openness, Help-seeking Propensity, and Indifference to Stigma. The total help-seeking score is calculated as a sum of the subscale totals with higher scores indicate more favourable attitudes towards helpseeking [30].

Psychological distress A 6-item self-administered Kessler psychological distress scale (K6) with good internal consistency (Cronbach's alpha $=0.84$ ) will be used. The total score ranges from 0 to 24 with a higher score indicating higher distress [31].

Resilience A 25-item Connor-Davidson Resilience Scale (CD-RISC) will be used. The CD-RISC is a valuable measure to assess resilience among adolescents in lowincome settings. Psychometric properties of the Kannada version of CD-RISC were established in a sample of adolescent girls from low-income settings. The total score ranges from 0 to 100 , with higher scores reflecting greater resilience [32].

A semi-structured interview schedule will measure socio-demographic details and contain case vignettes/ questions to assess MH-FA knowledge and intentions to provide MH-FA (proxy measures of behaviour change).

Exploratory outcomes A compelling body of evidence points to the potential influence of SUMS intervention on a range of adolescent health-related outcomes such as academic achievement, absenteeism rate, educational stress, suicidal ideation, bullying and substance use, which will be explored.

Pre-testing of study instruments The pre-validated study instruments will be translated into local language and back-translated for conceptual equivalence. For pretesting, a sample size of 42 is estimated to establish reliability of at least 0.85 , against acceptable value of 0.7 (with $\alpha=0.05 ; \beta=0.20$ ) for a minimum of 6 items [33]. The translated study instruments will be pre-tested on a representative sample for cultural relevance and comprehensibility and validated for internal consistency before their final inclusion. The final study instrument administered will be bilingual.

Sample size and power Positive mental health literacy measured using the 10-item Mental Health-Promoting Knowledge (MHPK-10) scale is considered primary outcome for the present study [26]. However, the IntraCluster Correlation for MHPK-10 score is unknown. Based on evidence from similar adolescents studies, we assumed a conservative and modest estimate of ICC $(\rho)$ between 0.01 and 0.05 [34, 35]. From the Kolar District
Report (as on 30/3/2016), an estimated average of 50 students is expected from grade- 6 and 7 of each higherprimary schools. With a need for 4 schools per arm with 50 students per school, we powered the trial to be able to detect a standardised difference (difference in means/ $\mathrm{SD}$ ) of 0.37-0.56 (medium effect size) for MHPK-10 scores between intervention and control groups for a two-sided level of significance at 5\% $(\alpha=0.05)$ with $85 \%$ power. For the above assumption, the design effect varied from 1.5 to 3.5 for ICC $(\rho)$ values from 0.01 to 0.05 , respectively [36]. We expect a minimum sample size of 400 across 8 schools for $85 \%$ power. Even with a $5 \%$ loss to follow-up, the study will still have more than $80 \%$ power.

Randomisation From the eligible higher-primary school-list obtained from the education department of Srinivaspura taluka, eight schools will be randomly selected using computer-generated random-number by an independent researcher. The selected schools will then be assigned to the intervention, and waitlist-control condition (receiving intervention after the post-assessment in intervention-group) by the independent researcher through the random-allocation sequence. To ensure allocation concealment, randomly selected schools will be assigned unique study numbers that will not be known to the independent researcher at the central office. The sequence will be delivered through sealed, opaque envelopes.

The selected-schools will be invited to participate in the study, and briefing-meetings will be conducted to inform principals of selected-schools regarding the study. Information on any initiatives/activities related to target outcomes in the selected-schools will be obtained. Local consortium and local stakeholders for education will be interacted to ensure minimal refusal of the selected schools. In case of refusal, the refusing school will be replaced with another school from the eligible school-list. A due care will be taken to prevent possible contamination so that no control-schools will be selected within $5 \mathrm{~km}$ radius of intervention-schools. An alternate covariate-constrained randomisation will be considered only if we do not reach an acceptable baseline balance through simple-randomisation.

Blinding Given the nature and complexity of intervention \& its delivery, it will not be possible to completely blind or conceal the intervention from the teachers, students, and field-staff. The trial will be open-label. Nonetheless, the study-statistician will be blinded till the database is locked for final-analysis.

Student-recruitment Enrolment will start after obtaining assent and parental-consent from the eligible 
students. In the case of multiple sections with $>60$ students in each grade, one section will be randomly selected from each grade. The intervention will be provided to all students in the selected grades irrespective of their number. The teachers will be free to provide intervention for other sections, but data will not be collected.

Intervention-schools For the schools randomised to the intervention arm, the teachers nominated by their designated superior officials will be trained on the curriculum resource guide content in a 3-day workshop. The teachers who provide consent to participate in the research, complete the training, stay in the selectedschools for the study period and deliver the intervention will be selected. Following baseline assessment of participating students, the trained teachers will then proceed with the implementation of SUMS Intervention Curriculum Resource Guide, which requires approximately 10$15 \mathrm{~h}$ of classroom time over two months. In the intervention delivery, the teachers will be given pedagogical flexibility but will be instructed to adhere to the standardised core content, recommended lesson plans and procedures to maintain fidelity (Table 2). Booster sessions will be provided at 2-months and 5-months following the initial intervention.

Control-schools Control-schools will continue to provide regular teaching as usual of the existing course to their students. The control group will receive the interventions at the end of the 12-month follow-up assessment in intervention-schools. The teachers in the waitlist control arm will be trained just one month before their scheduled intervention to prevent contamination.

Parental-consent An information letter detailing the intervention program and data collection along with consent-form will be sent to the parents/guardians of participating class for parental-consent. Parental-consent will be recorded with a signature/thumbprint. Existing school communication channels (letter, diary or parent's teachers meeting) will be used to maximise the parentalconsent for child participation. Parents will be provided an opportunity to contact the field-team to discuss the study and their participation. An attempt will be made to meet the non-responding parents to obtain their consent. Children without parental consent in the selected class will be permitted to attend intervention sessions, but data will not be collected from them.

Discontinuation-criteria The students may withdraw their participation at any point in time during the study. An attempt will be made to document the reasons for withdrawal.
Table 2 Quality assurance and data management

Field-team: The field-team will comprise of Project-Assistant who will be trained in standard protocols of questionnaire administration for data collection, coordinate with local stakeholders, monitor compliance of intervention protocol and its deviations and also conduct publicengagement activities.

Post-recruitment retention strategies: For the Project-Assistant, all the activities required to achieve the trial goals will be communicated clearly. A weekly meeting will be held to sort out the field related issues. In addition, the teachers, principals, and other key stakeholders will be informed on the trial's status and progress through newsletters.

Trial-Monitoring: The Project-Assistant will check for interventiondelivery, protocol-adherence, data-collection and reporting using supervisory check-list during their monthly visits. Principal-Investigator will review this monthly visit reports during monthly review meetings. The central-research team (comprising of Principal-Investigator and other expert-collaborators) will have meetings once in 6 months at NIMHANS to review the progress, work plan, discuss and troubleshoot any unresolved field issues. A register will be maintained for all review meetings. Constant support will be obtained from the expert collaborators through tele-meetings.

A Trial Steering Committee (TSC) and Data Safety Monitoring Board (DSMB) will be constituted independently of the research team, the members of whom will have no conflict of interest. The TSC will comprise experienced experts to oversee the trial and independently evaluate the trial to monitor progress, intervention delivery, protocol adherence and data management. A three-member DSMB will monitor the data as the trial progresses to identify any emerging safety concerns for the study participants and problems in the study's conduct. DSMB will decide on the continuation of trial following interim analysis.

Intervention Fidelity: Teachers will be encouraged to fill a protocoladherence checklist. The educational-intervention delivered by the teacher will be audio-recorded to assess fidelity of intervention delivery. A random sample of audiotaped sessions will be evaluated for each teacher using fidelity checklists for intervention-delivery based on the standardised core content of the SUMS intervention curriculum resource guide. Feedback will be provided to support the booster-training sessions. Our fidelity assessment will also incorporate feedback and recommendations of the independent evaluation and reports of supervisorychecklist.

Data Management: The study will have meaningful engagement with key stakeholders across all study phases. All data stored securely in-line with our data-management protocol will protect the confidentiality of participants. Any personal identifiers of study participants will be removed before analysis. Audiotapes and transcripts containing participants identity (if any) will be destroyed post-analysis of data.

Participant's identity will not be revealed in any of the publications related to this research. The principal investigator will have access to the final trial dataset.

Timing of outcome-measurement Data will be collected using self-administered questionnaire. Students will complete the survey during class time in the presence of trained project assistant/ teacher to assist the children with reading, writing and answering any queries. Data will be collected at 4-timepoints from both intervention and control schools: baseline and 6-weeks, 6-months and 12-months post-intervention (Fig. 2). To maximise data completeness, all effort will be made to obtain data from absentee students through liaison with key school-staff.

Adverse-events We do not anticipate any risk to the participating students due to our interventions. 


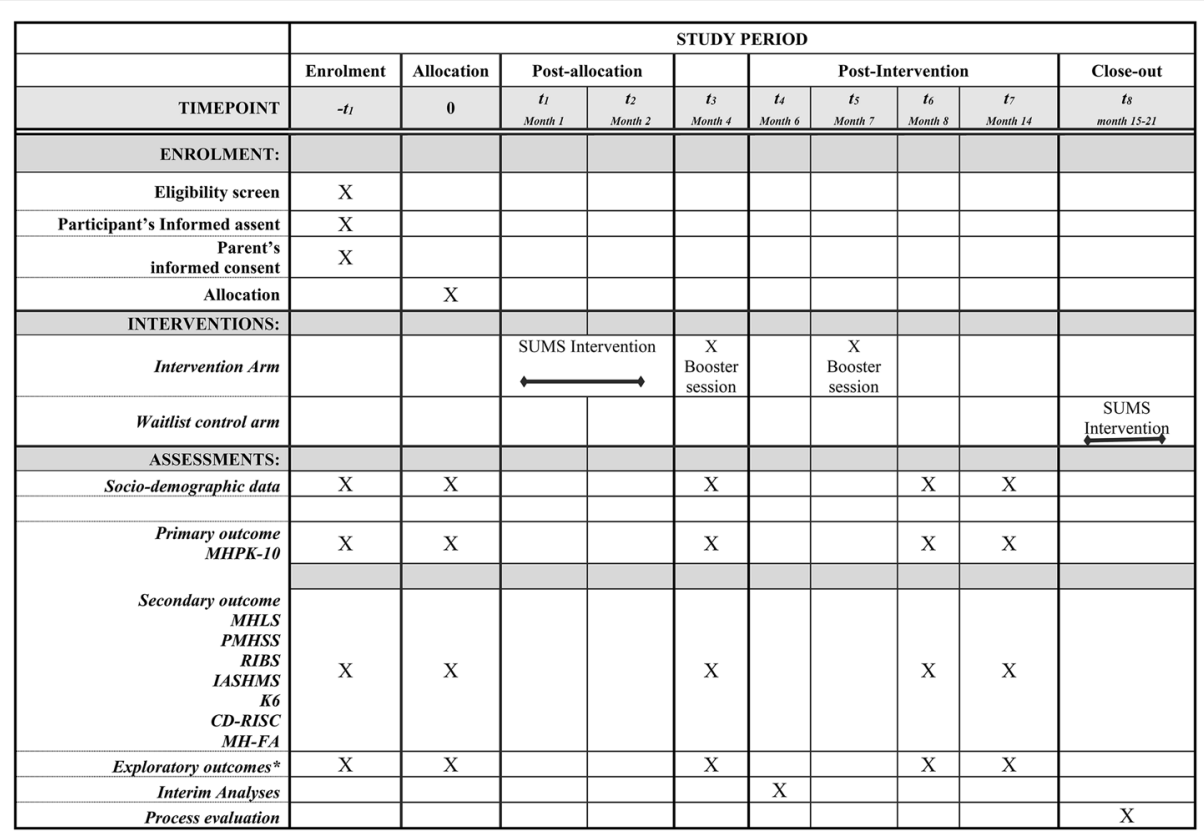

Fig. 2 Trial process. MHPK: Mental Health Promoting Knowledge; MHLS: Mental Health Literacy Scale; PMHSS: Peer Mental Health Stigmatization Scale; RIBS: Reported and Intended Behaviour Scale; IASMHS: Inventory of Attitudes towards Seeking Mental Health Services; K6: Kessler psychological distress scale; CD-RISC: Connor-Davidson Resilience Scale; MH-FA: First Aid in Mental Health. *Exploratory outcomes include academic achievement, absenteeism, educational stress, suicidality, bullying and substance use

However, potential discomfort due to sensitive questions of survey assessment related to self-perception and wellbeing cannot be ruled out when answering in the classroom. Due-care will be taken to ensure the privacy and confidentiality of students while filling the survey forms. A register \& form will be maintained to record unexpected severe events during the trial. Any students who require professional mental help during the study will be referred to the district mental health team for further management.

Process evaluation The process evaluation will include concurrent fidelity assessment and a qualitative study (focus group/in-depth interviews) among students/ teachers during the final stages of the trial to explore the implementation of the intervention and identify contextual factors associated with outcomes. Teachers will be provided with questionnaires to evaluate the Curriculum Guide and to provide feedback on their experience.

\section{Plan of analysis}

The study tools/measures will be examined for internal consistency. Demographic and baseline characteristics will be summarised using descriptive statistics. Principalanalyses will be intention-to-treat which will take into account clustering by the school and repeated measures. To assess the effectiveness of interventions, outcomes will be compared between two trial arms using linearmixed-model analysis for continuous outcomes and generalised linear mixed models or generalised estimating evaluation (GEE) for binary outcomes. The analysis will include all participants with at least one outcome measurement at 6 weeks post-intervention. A perprotocol analysis will also be conducted along with intention-to-treat analysis to examine the influence of missing data patterns. Multiple imputation will be attempted for missing values. The analysis will be adjusted for potential confounders that are identified apriori during the study. Subgroup analysis will be performed for important socio-demographic variables. Statistical significance will be set at $p$-value $<0.05$, and Bonferroni's correction will be made for multiple testing (if any).

For qualitative analysis, all the verbatims of interviews/ group discussions will be transcribed and translated into English for thematic analysis.

Interim-analyses Interim analyses is planned for the follow-up data collected at 6-weeks post-intervention and will be submitted to Data Safety \& Monitoring Board (DSMB) for further review (Table 2).

Stopping-rule The DSMB will be asked to advise stopping the trial if they have proof beyond doubt that intervention is futile. The trial's stopping-rule will be based on evidence-of-futility in $>70 \%$ of outcome measures (both primary \&secondary) at interim-analyses and critical feedback from the local stakeholders. 


\section{Trial status and review}

The trial was reviewed and funded by Indian Council of Medical Research (Id: 2017-4697 version F1 dated 02 September 2019). The trial was reviewed and approved by the Institute Ethics Committee, NIMHANS (No.NIMHANS $/ 19^{\text {th }}$ IEC (BS\&NS DIV.)/2019 on 2019-07-17. The trial was registered at Clinical Trials Registry-India with identification code: CTRI/2019/07/020394 dated 2019-07-29. Due to COVID-19 Pandemic and school closures, recruitment for the trial is anticipated after March 2022.

\section{Discussion}

This pragmatic, cluster randomised waitlist-controlled design will examine the effectiveness of classroom-based teacher-led integrated school mental health intervention in improving school-going adolescent children's mental health and well-being. To the best of our knowledge, this is the first study to integrate MHL with MHP and MHFA for promoting mental health well-being among adolescent school children in India.

The trial is an extension of a series of focused research activities undertaken in Child and Adolescent Mental Health (CAMH) in Kolar district, Karnataka. The activities included situational analysis, numerous consultation workshops with teachers and various training programmes organised in-collaboration with the education department in Kolar. Following interactive discussions with NIMHANS experts and a national-level symposium, a road-map and draft action plan for integrated CAMH services in a district was finalised. The present trial is a part and extension of the action plan developed for promoting CAMH. Though the mental health intervention domains (MHP+ MHL + MH-FA) are closely interlinked with considerable cross-cutting areas of action, we intentionally compartmentalised it for better focus and delivery. With a need to build a more substantial evidence base on effective School Mental Health Promotion approaches in low-income countries, the study builds on the facilitating role of school climate in promoting mental health and related outcomes among adolescents and evaluates its effectiveness through a robust and systematic methodology.

\section{Limitations}

As cultural aspects are likely to influence the effectiveness of SUMS interventions, the trial results should be interpreted within the given cultural context. As this study is being conducted in a relatively small geographical area, a larger multi-centre trial is required to better understand SUMS interventions' replicability and scalability, especially in different cultural settings.

India has recently launched the Health and Wellness Ambassador initiative under the School Health
Programme of Ayushman Bharat Mission [37]. This initiative trains two identified teachers from each school to facilitate the healthy survival of school children under 11 themes, including emotional well-being and mental health. In this context, the evidence obtained from this study will have implications for implementing and operationalising the Health and Wellness Ambassador initiative in schools. Further, the barriers and challenges identified in the present study will provide valuable inputs to improve school climate for promoting mental health among school-going adolescents, especially in resource-poor settings. This study will contribute significantly towards transforming the vision of shifting from "survival to healthy survival" of adolescent children in developing countries into reality.

\section{Abbreviations \\ CAMH: Child and Adolescent Mental Health; CD-RISC: Connor-Davidson Resilience Scale; DSMB: Data Safety \& Monitoring Board; HPS: Health Promoting Schools; IASMHS: Inventory of Attitudes towards Seeking Mental Health Services; KIl: Key informant interview; MH-FA: First Aid in Mental Health; MHL: Mental Health Literacy; MHLS: Mental Health Literacy Scale; MHP: Mental Health Promotion; MHPK: Mental Health Promoting Knowledge; NIMHANS: National Institute of Mental Health And Neuro Sciences; PMHS S: Peer Mental Health Stigmatization Scale; RE-AIM: Reach, Effectiveness, Adoption, Implementation, and Maintenance; RIBS: Reported and Intended Behaviour Scale; SUMS: Scaling Up of Mental health in Schools; TSC: Trial Steering Committee}

\section{Acknowledgements}

We are grateful to the Department of Education, Kolar district, Government of Karnataka for their support and cooperation. We also gratefully acknowledge the Research Methodology Cell and Division of Reproductive Biology, Maternal and Child Health, Indian Council of Medical Research for their support and coordination. We thank Dr. Ashoo Grover, Indian Council of Medical Research for her support and guidance.

\section{Authors' contributions}

$\mathrm{SA}$ and $\mathrm{KJ}$ formulated the research question, the conception and design of the study and conducted the grant application. KM defined the statistical methods; NPM, ES, PM, ADB, HKT, AA and SV support the manual development, intervention delivery and drafting the manuscript. GNR provided administrative support in the coordination of the study, ethics approval and drafting of the manuscript. All authors revised the manuscript for important intellectual content and approved the final submitted version of the manuscript.

\section{Funding}

Financial support for this project is granted by the Indian Council of Medical Research, New Delhi, India under "Call for concept proposals for identifying Young and Middle level Faculty to participate in Research Methodology Workshop". Funding agency was involved in reviewing the protocol. Funding agency shall carry out reviewing of yearly progress and auditing. The funding agency will have no role in the study design, data collection, data analysis, data interpretation, or writing of the report. Project results shall be presented to the funding agency which will review the results in terms of meeting up the project objectives.

\section{Availability of data and materials}

Data sharing is not applicable to this article as no datasets have yet been generated or analyzed.

\section{Declarations}

Ethics approval and consent to participate

The trial was reviewed and approved by NIMHANS Ethics Committee [(No.NIMHANS/19 $9^{\text {th }}$ IEC (BS\&NS DIV.)/2019], National Institute of Mental Health And Neuro Sciences (NIMHANS), Bangalore, India on 2019-07-17. The trial 
was registered at Clinical Trials Registry- India with identification code: CTRI/ 2019/07/020394 dated 2019-07-29. Informed assent from the participating children and written informed consent from the participating children's parents/guardians will be obtained before any study procedure. Appropriate permissions will be obtained from the relevant district education authority and school principals for conducting the study. Written informed consent will be obtained from the teachers for their participation in this research.

\section{Consent for publication}

Not Applicable.

\section{Competing interests}

The authors declare that they have no competing interests.

\section{Author details}

${ }^{1}$ Department of Epidemiology, National Institute of Mental Health And Neuro Sciences, Bangalore, Karnataka, India. ${ }^{2}$ Department of Psychiatric Social Work, National Institute of Mental Health And Neuro Sciences, Bangalore, Karnataka, India. ${ }^{3}$ Department of Biostatistics, All India Institute of Medical Sciences, New Delhi, Delhi, India. ${ }^{4}$ Clinical Psychologist, Independent Consultant, Bangalore, Karnataka, India. ${ }^{5}$ Department of Child and Adolescent Psychiatry, National Institute of Mental Health And Neuro Sciences, Bangalore, Karnataka, India.

Received: 13 October 2021 Accepted: 22 October 2021 Published online: 06 November 2021

\section{References}

1. Kieling C, Baker-Henningham H, Belfer M, Conti G, Ertem I, Omigbodun O, et al. Child and adolescent mental health worldwide: evidence for action. Lancet. 2011;378(9801):1515-25. https://doi.org/10.1016/S0140-6736(11)60827-1.

2. American Psychological Association. Developing adolescents: a reference for professionals. 2002. http://www.apa.org/pi/families/resources/develop.pdf. Accessed 14 Jan 2019

3. $\mathrm{WHO} \mid$ Adolescent health epidemiology. WHO. https://www.who.int/ma ternal_child_adolescent/epidemiology/adolescence/en/. Accessed 14 Jan 2019.

4. Amudhan S, Gururaj G, Varghese M, Benegal V, Rao GN, Sheehan DV, et al. A population-based analysis of suicidality and its correlates: findings from the National Mental Health Survey of India, 2015-16. Lancet Psychiatry. 2020; 7(1):41-51. https://doi.org/10.1016/S2215-0366(19)30404-3.

5. Gulliver A, Griffiths KM, Christensen H. Perceived barriers and facilitators to mental health help-seeking in young people: a systematic review. BMC Psychiatry. 2010;10(1):113. https://doi.org/10.1186/1471-244X-10-113.

6. Evans-Lacko S, Brohan E, Mojtabai R, Thornicroft G. Association between public views of mental illness and self-stigma among individuals with mental illness in 14 European countries. Psychol Med. 2012;42(8):1741-52. https://doi.org/10.1017/S0033291711002558.

7. Rüsch N, Evans-Lacko SE, Henderson C, Flach C, Thornicroft G. Knowledge and attitudes as predictors of intentions to seek help for and disclose a mental illness. Psychiatr Serv. 2011 Jun;62(6):675-8. https://doi.org/10.1176/ ps.62.6.pss6206_0675.

8. Kutcher S, Bagnell A, Wei Y. Mental health literacy in secondary schools: a Canadian approach. Child Adolesc Psychiatr Clin N Am. 2015;24(2):233-44. https://doi.org/10.1016/j.chc.2014.11.007.

9. Bonabi H, Müller M, Ajdacic-Gross V, Eisele J, Rodgers S, Seifritz E, et al. Mental health literacy, attitudes to help seeking, and perceived need as predictors of mental health service use: a longitudinal study. J Nerv Ment Dis. 2016;204(4):321-4. https://doi.org/10.1097/NMD.0000000000000488.

10. Gulliver A, Griffiths KM, Christensen H, Brewer JL. A systematic review of help-seeking interventions for depression, anxiety and general psychological distress. BMC Psychiatry. 2012;12(1):81. https://doi.org/10.1186/1471-244 $\mathrm{X}-12-81$.

11. Jorm AF, Korten AE, Jacomb PA, Christensen H, Rodgers B, Pollitt P. "Mental health literacy": a survey of the public's ability to recognise mental disorders and their beliefs about the effectiveness of treatment. Med J Aust. 1997; 166(4):182-6. https://doi.org/10.5694/j.1326-5377.1997.tb140071.x.

12. Lam LT. Mental health literacy and mental health status in adolescents: a population-based survey. Child Adolesc Psychiatry Ment Health. 2014;8(1):26. https://doi.org/10.1186/1753-2000-8-26.
13. Kutcher S, Wei Y, Costa S, Gusmão R, Skokauskas N, Sourander A. Enhancing mental health literacy in young people. Eur Child Adolesc Psychiatry. 2016; 25(6):567-9. https://doi.org/10.1007/s00787-016-0867-9.

14. Hart LM, Mason RJ, Kelly CM, Cvetkovski S, Jorm AF. 'teen Mental Health First Aid': a description of the program and an initial evaluation. Int J Ment Health Syst. 2016;10:3.

15. Chisholm K, Patterson P, Torgerson C, Turner E, Jenkinson D, Birchwood M. Impact of contact on adolescents' mental health literacy and stigma: the SchoolSpace cluster randomised controlled trial. BMJ Open. 2016;6(2): e009435. https://doi.org/10.1136/bmjopen-2015-009435.

16. Kitchener B, Jorm A, Kelly C. Mental health first aid manual. 3rd ed. Melbourne: Mental Health First Aid Australia; 2013.

17. Perry Y, Petrie K, Buckley H, Cavanagh L, Clarke D, Winslade M, et al. Effects of a classroom-based educational resource on adolescent mental health literacy: a cluster randomised controlled trial. J Adolesc. 2014;37(7):1143-51. https://doi.org/10.1016/j.adolescence.2014.08.001.

18. Langford R, Bonell C, Komro K, Murphy S, Magnus D, Waters E, et al. The health promoting schools framework: known unknowns and an agenda for future research. Health Educ Behav. 2017;44(3):463-75. https://doi.org/10.11 77/1090198116673800.

19. Mũkoma W, Flisher AJ. Evaluations of health promoting schools: a review of nine studies. Health Promot Int. 2004;19(3):357-68. https://doi.org/10.1093/ heapro/dah309.

20. Pinto-Foltz MD, Logsdon MC, Myers JA. Feasibility, acceptability, and initial efficacy of a knowledge-contact program to reduce mental illness stigma and improve mental health literacy in adolescents. Soc Sci Med. 2011;72(12): 2011-9. https://doi.org/10.1016/j.socscimed.2011.04.006.

21. Census of India Website: Office of the Registrar General \& Census Commissioner, India. http://www.censusindia.gov.in/2011census/C-series/ C-13.html. Accessed 18 May 2018.

22. RKSK (Rashtriya Kishor Swasthya Karyakram) - Strategy Handbook. Ministry of Health and Family Welfare, Government of India, New Delhi, India; 2014 http://nhm.gov.in/images/pdf/programmes/rksk-strategy-handbook.pdf. Accessed 25 Jun 2018.

23. Patton GC, Glover S, Bond L, Butler H, Godfrey C, Di Pietro G, et al. The gatehouse project: a systematic approach to mental health promotion in secondary schools. Aust N Z J Psychiatry. 2000;34(4):586-93. https://doi. org/10.1080/j.1440-1614.2000.00718.x.

24. Kutcher S, Wei Y, Hashish M. Chapter 8 - mental health literacy for students and teachers: a "school friendly" approach. In: Hodes M, Gau S, editors. Positive mental health, fighting stigma and promoting resiliency for children and adolescents. San Diego: Academic Press; 2016. p. 161-72. https://doi.org/10.1016/B978-0-12-804394-3.00008-5.

25. Weiner BJ, Lewis CC, Stanick C, Powell BJ, Dorsey CN, Clary AS, et al. Psychometric assessment of three newly developed implementation outcome measures. Implement Sci. 2017;12(1):108. https://doi.org/10.1186/ s13012-017-0635-3.

26. Bjørnsen HN, Eilertsen MB, Ringdal R, Espnes GA, Moksnes UK. Positive mental health literacy: development and validation of a measure among Norwegian adolescents. BMC Public Health. 2017;17(1):717. https://doi.org/1 0.1186/s12889-017-4733-6.

27. O'Connor M, Casey L. The mental health literacy scale (MHLS): a new scalebased measure of mental health literacy. Psychiatry Res. 2015;229(1-2):5116. https://doi.org/10.1016/j.psychres.2015.05.064.

28. McKeague L, Hennessy E, O'Driscoll C, Heary C. Peer mental health stigmatization scale: psychometric properties of a questionnaire for children and adolescents. Child Adolesc Ment Health. 2015;20(3):163-70. https://doi. org/10.1111/camh.12088.

29. Evans-Lacko S, Rose D, Little K, Flach C, Rhydderch D, Henderson C, et al. Development and psychometric properties of the reported and intended behaviour scale (RIBS): a stigma-related behaviour measure. Epidemiol Psychiatr Sci. 2011;20(3):263-71. https://doi.org/10.1017/S2045796011000308.

30. Mackenzie CS, Knox VJ, Gekoski WL, Macaulay HL. An adaptation and extension of the attitudes toward seeking professional psychological help scale. J Appl Soc Psychol. 2004;34(11):2410-33. https://doi.org/10.1111/j.1 559-1816.2004.tb01984.x.

31. Mewton L, Kessler RC, Slade T, Hobbs MJ, Brownhill L, Birrell L, et al. The psychometric properties of the Kessler psychological distress scale (K6) in a general population sample of adolescents. Psychol Assess. 2016;28(10): 1232-42. https://doi.org/10.1037/pas0000239. 
32. Sidheek KPF, Satyanarayana VA, Sowmya HR, Chandra PS. Using the Kannada version of the Connor Davidson resilience scale to assess resilience and its relationship with psychological distress among adolescent girls in Bangalore. India Asian J Psychiatr. 2017;30:169-72. https://doi.org/10.1016/j.a jp.2017.10.015.

33. Bonett DG. Sample size requirements for estimating intraclass correlations with desired precision. Stat Med. 2002;21(9):1331-5. https://doi.org/10.1002/ sim.1108.

34. Smedegaard S, Christiansen LB, Lund-Cramer P, Bredahl T, Skovgaard T. Improving the well-being of children and youths: a randomized multicomponent, school-based, physical activity intervention. BMC Public Health. 2016;16(1):1127. https://doi.org/10.1186/s12889-016-3794-2.

35. Kuyken W, Nuthall E, Byford S, Crane C, Dalgleish T, Ford T, et al. The effectiveness and cost-effectiveness of a mindfulness training programme in schools compared with normal school provision (MYRIAD): study protocol for a randomised controlled trial. Trials. 2017;18(1):194. https://doi.org/10.11 86/s13063-017-1917-4.

36. Donner A, Birkett N, Buck C. Randomization by cluster. Sample size requirements and analysis. Am J Epidemiol. 1981;114(6):906-14. https://doi. org/10.1093/oxfordjournals.aje.a113261.

37. Press Information Bureau, Government of India. Curriculum for School Health Ambassador Initiative under Ayushman Bharat. https://pib.gov.in/ newsite/PrintRelease.aspx?relid=199289. Accessed 7 Aug 2020.

\section{Publisher's Note}

Springer Nature remains neutral with regard to jurisdictional claims in published maps and institutional affiliations.

Ready to submit your research? Choose BMC and benefit from:

- fast, convenient online submission

- thorough peer review by experienced researchers in your field

- rapid publication on acceptance

- support for research data, including large and complex data types

- gold Open Access which fosters wider collaboration and increased citations

- maximum visibility for your research: over $100 \mathrm{M}$ website views per year

At $\mathrm{BMC}$, research is always in progress.

Learn more biomedcentral.com/submissions 